\title{
A importância estratégica da informação em saúde para o exercício do controle social
}

\author{
The strategic importance of health information for social control
}

\author{
Alessandra Ximenes da Silva ${ }^{1}$ \\ Eliane Aparecida $\mathrm{Cruz}^{1}$ \\ Verbena M elo ${ }^{1}$
}

${ }^{1}$ Secretaria Executiva do

Conselho Nacional de

Saúde. Esplanada dos

Ministérios, Bloco "G" -

Edifício Anexo, Ala"B"/104

70.058-900 Brasília DF.

alessandra.ximenes@saude.

gov.br

\begin{abstract}
This paper discusses the relationship between the State and Brazilian society since the early 1980s. Particularly noteworthy are the $\mathrm{H}$ ealthcare Councils that draw up strategies and controls for implementing $\mathrm{H}$ ealthcare Policies. It also analyzes healthcare information policies for deploying social controls and helps extend theaccess of these Councils to these policies at various levels of complexity, based on the principle of allround healthcare. Brazil's National H ealth Council has contributed to this process through its Intersector Healthcare Communication and Information Commission, which was brought back into operation in 2005. To do so, Decentralized Regional Seminars were held, as well as a $\mathrm{Na}$ tional Seminar, discussing the construction of a Pact for the Democratization and Quality of $\mathrm{H}$ ealth Communication and Information. The proposals presented by the $\mathrm{H}$ ealth Councils were analyzed, with the outstanding being the establishment of indicators to evaluate and oversee information quality and publicizing the rights of theusers of Brazil's N ational Health System (SUS). Key words Social control, Health information, Democratization
\end{abstract}

Resumo 0 artigo trata da nova forma de relacionamento entre Estado e sociedade a partir da década de 80, na realidade brasileira. Destacamse os consel hos de saúde queatuam na formulação de estratégias e no controle da execução da Política de Saúde. Analisa as Políticas de Informação em saúde para o exercício do controle social e contribui para ampliar o acesso dos consel hos de saúde a essas políticas em seus diferentes níveis de complexidade, à luz do princípio da integralidade e da atenção à saúde. 0 Conselho Nacional de Saúde tem contribuído nesse processo através de sua Comissão I ntersetorial de Comunicação e Informação em Saúde, que foi reativada em 2005. Para tanto, foram realizados Seminários Regionais Descentralizados e um Seminário Nacional para discutir a construção do Pacto pela Democratização e Qualidade da Comuni cação e Informação em Saúde. Foram analisadas as propostas apresentadas pelos conselhos de saúde, entre as quais destacam-se: estabelecer indicadores para avaliar/acompanhar a qualidade da informação e publicizar os direitos dos usuários no SUS.

Palavras-chave Controle social, Informação em saúde, Democratização 


\section{Introdução}

0 artigo trata da nova forma de relacionamento entre Estado/sociedade, configurada através dos canais institucionais de participação, enquanto espaço de decisão e de negociação de interesses, na gestão da Política deSaúde, no que diz respeito ao processo de construção da Política de Informação e Comunicação em Saúde.

0 nosso interesse por esta temática corresponde às mudanças ocorridas na relação Estado/sociedade, vinculando-se com o movimento mais geral da sociedade brasileira na década de 80 , quando se intensificaram as propostas de democratização da sociedade, na qual seinscreve a da gestão das políticas públicas. Busca-se com a democratização eliminar as formas autoritárias e tradicionais de gestão das Políticas Sociais, bem como a adoção de práticas que favoreçam uma maior transparência das informações e maior participação da sociedade no processo decisório.

Uma das propostas para a democratização é a implantação e o funcionamento dos conselhos de saúde. Os conselhos de saúde foram instituídos nas três esferas de governo federal, estadual e municipal. Segundo a legislação em vigor, as decisões emanadas desses órgãos devem tratar da formulação de estratégias e do controle da execução das políticas de saúde, com pleno acesso aos aspectos econômico-financeiros voltados para o setor, propondo critérios para a programação, a execução, 0 acompanhamento, a avaliação e, também, a elaboração e a aprovação do plano de saúde, bem como o estabelecimento de estratégias para a sua execução.

Algumas dificuldades são apresentadas quanto ao funcionamento dos consel hos de saúdeque exigem práticas democráticas, esbarrando em dificuldades relacionadas à inconsistência deuma cultura política democrática e à persistência de uma tradição autoritária, ainda presente na realidadenacional.

Ressaltamos, que na Seguridade Social, a Política de Saúde tem apresentado maiores avanços acerca do processo de descentralização político-administrativa. Com a promulgação das Leis $8.080 / 90^{1}$ e $8.142 / 90^{2}$, foi o primeiro setor em que houve a regulamentação e possui uma estrutura formada pelo Conselho $\mathrm{N}$ acional de Saúde, pelos Conselhos Estaduais em todos os estados brasileiros e Conselhos Municipais de Saúde na maioria dos 5.564 municípios brasileiros. Existem ainda as Conferências de Saúde e as Plenárias N acionais de Conselhos de Saúde (com o objetivo deser um fórum de articulação entre conselhos).

Segundo Chaui ${ }^{3}$, os sujeitos políticos participam da vida social em proporção ao volume e qualidade das informações que possuem em especial, a partir das suas possibilidade de acesso às fontes de informação e de condições favoráveis de aproveitamento delas, de forma a poderem intervir como produtores do saber.

A informação possui uma dimensão política e estratégica para os processos decisórios nos conselhos de saúde, fazendo-senecessário a existência de mecanismos participativos e democráticos. 0 aparato que envolve a informação em saúdenecessita estar a serviço dos sujeitos políticos; sendo assim, 0 acesso às informações precisa ser democratizado e as barreiras existentes, superadas. Para tanto, na perspectiva de fortalecer o exercício do controle social na política de saúde, se faz necessário, por parte dos sujeitos políticos envolvidos no processo, o efetivo conhecimento do Sistema Ú nico de Saúde, da Legislação, da realidade epidemiológica, assistencial, financeira, política, etc. para acompanhar, estimular e avaliar o sistema de informações em saúde no nível municipal, estadual e nacional.

\section{Pacto pela democratização e qualidade da informação e comunicação em saúde}

A 12a Conferência Nacional de Saúde ${ }^{4}$ discutiu e avaliou a necessidade de elaboração e implementação de uma política de informação, comunicação e informática para o Sistema Ú nico de Saúde-SUS e, conseqüentemente, para o controle social. Essa política deve estar pautada nas diretrizes do SUS, na política de saúde vigentee, também, na garantia das ações e dos recursos que permitam a democratização do acesso à informação, comunicação e informática em saúde. Essa discussão trata da possi bilidade da geração de novos processos, produtos e de mudanças nos model os institucionais de gestão.

A expressão controle social tanto é empregada para designar o controle do Estado sobre a sociedade, quanto para designar o controle da sociedade sobre as ações do Estado. E, mesmo nestas duas acepções, existem diferenças devido ao entendimento da função do Estado e da extensão do seu poder e/ou devido à forma como se concebe a sociedade civil. N esta segunda perspectiva, a temática do controlesocial tomou vulto no Brasil a partir do processo de democratização na década de 1980 e, principalmente, com 
a institucional ização dos mecanismos de participação nas políticas públicas na Constituição de 1988 e nas leis orgânicas posteriores como instâncias colegiadas de caráter permanente e deliberativo. Esta participação adquiriu uma direção de controle social posta pelos setores progressistas da sociedade, ou seja, de controle por parte dos segmentos organizados da mesma sobre as ações do Estado no sentido deste, cada vez mais, atender aos interesses da maioria da população, em reverso ao período ditatorial de controle exclusivo do Estado sobre a sociedade, cerceando qualquer expressão dessa.

I sso demonstra a importância atribuída à informação em saúdeeo controlesocial na constituição e funcionamento do Sistema Único de Saúde. Tanto um conceito quanto o outro são pouco problematizados, mas são conceitos relevantes e estratégicos.

O bserva-se, atualmente, que tem crescido a divulgação de informações e dados envolvendo doenças, pesquisas, diagnósticos, entre outros, pelos mei os de comunicação, o quejá caracteriza um grande avanço na disseminação da informação em saúde. 0 grande desafio para o setor é 0 de tornar essa informação acessível aos usuários do SUS de maneira universal. Ou seja, não basta divulgar os dados pura e simplesmente, é necessário apresentar a informação numa linguagem adequada, de forma a permitir que qualquer sujeito possa se apropriar do conhecimento. Em outras palavras, um dos maiores avanços da saúde pública brasileira será quando pudermos socializar dados, pesquisas e informações através dos diferentes meios de comunicação (internet, jornais, revistas, rádio, TV, etc.) para a população usuária do SUS, de forma ágil e clara.

A informação e a comunicação em saúde podem atender e promover avanços significativos nas novas relações propostas a partir da criação dos conselhos de saúde e do novo cenário nacional que se constituiu com a participação popular na tomada de decisão nas três esferas. Essas duas áreas devem priorizar suas definições e suas ações com iniciativas que atendam aos usuários do SUS na atenção básica, média e alta complexidade, deforma a contribuir com novos paradigmas e conceitos quepossibilitem um avanço no sistema público de saúde brasileiro e respeitem os princípios fundamentais do SUS.

Segundo $\mathrm{Cardoso}^{5}$, a comunicação vem assumindo importância crescente na sociedade contemporânea. A facemais visível desse processo talvez seja o acel erado desenvolvimento detecnologias de informação e comunicação observa- do nas últimas décadas. Tal fato traz alguns desafios para o exercício do controlesocial, na perspectiva de superar a visão instrumental da comunicação e as práticas campanhistas; efetivar a descentralização e democratizar o acesso às tecnologias de comunicação.

No entanto, de acordo com Oliveira ${ }^{6}$, hoje a prática dos consel hos de saúde, em nível municipal, mereceuma avaliação mais crítica ecapaz de apontar os desafios e as contradições em relação ao controle público e a participação popular no Sistema Ú nico de Saúde, tendo como enfoque a questão da comunicação social.

O Conselho Nacional de Saúde, instância máxima de deliberação do SUS, vem pautando, nos últimos anos, discussões de temáticas sobre a informação e comunicação em saúde. As Conferências Nacionais de Saúde $10^{\underline{a} 7}$ e $11^{\underline{a}}$ discutiram eapontaram a necessidade de discussão mais aprofundada do tema. $\mathrm{Na}$ 12a Conferência $\mathrm{N}$ acional de Saúde, a informação e comunicação em Saúde foram discutidas em um eixo próprio, 0 que permitiu apontar propostas importantes para a área e iniciar uma discussão mais ampla sobre a questão.

No eixo $X$ da 12 a Conferência Nacional de Saúde, ficou definido que o Conselho $\mathrm{N}$ acional deSaúde deverá definir estratégias para deliberação e implementação de políticas articuladas de informação e comunicação na perspectiva dedar maior visibilidade para as diretrizes do SUS, a Política de Saúde, as ações e a utilização de recursos no sentido de ampliar a participação eo controle social. Para tanto, necessário se faz: 1) democratizar a informação e comunicação, em todos os aspectos; 2) viabilizar a rede de informação em saúde de âmbito nacional e; 3) desenvolver projetos nas três esferas de governo para garantir a divulgação permanente para a população, de decisões e informações dos conselhos e conferências de saúde.

Para tanto, o Conselho Nacional de Saúde, através da Comissão Intersetorial de Comunicação el nformação em Saúde 9 , vem imprimindo ações na perspectiva de discutir o Pacto pela $\mathrm{De}$ mocratização e Qualidade da Comunicação e Informação em Saúde. Foram realizados seis seminários descentralizados nas cinco regiões do país e um seminário nacional, que teve como objetivo discutir a Política de Comunicação, Informação e Informática em Saúde. D entre as ações, destacam-se a implementação do Cadastro Nacional de Conselhos de Saúde com informações dos consel hos estaduais e municipais para fortalecer a interlocução contínua entre os con- 
selhos de saúde; a criação do fique atento, um espaço de democratização da informação e comunicação em saúde a ser divulgado no jornal do CNS e outros meios de comunicação para tratar de temas que abranjam um problema/dimensão da saúde derelevância nacional quepossua impacto para a saúde da população e; projeto de inclusão digital no sentido de equipar e capacitar os conselhos de saúde para apropriação denovas tecnologiase conseqüentementemelhorar eter acesso à comunicação e informação em saúde.

De acordo com Moraes ${ }^{10}$, a demarcação de um escopo, comprometido com o Sistema Ú nico de Saúde, constitui-se em um dos principais desafios implícitos na formulação da agenda da Política Nacional de Informação, Informática e Comunicação em Saúde. Os diferentes interesses em disputa, sua vinculação com as novas tecnologias, a miríade das ações de informação e comunicação que repercutem em um emaranhado de setores da vida em sociedade, sua transversalidade, o maior ou menor uso e apropriação da informação por sujeitos políticos em seu agir estratégico, requerem cotidianamente escol has definidas a partir da correlação de forças políticas, sociais e econômicas em constante tensão.

0 desenvolvimento e incorporação da informação e comunicação e suas tecnologias de forma ampliada e disseminada pressupõem decisões políticas e econômicas que perpassam pelas relações de poder e produção do saber.

Alguns desafios são postos nesse debate para o exercício do controle social, os quais se destacam: a definição das informações relevantes (0 que informar?); o mecanismo de acesso mais adequado às diferentes situações dos Conselhos de Saúde (como informar?); como tratar a informação e a opção tecnológica a ser adotada.

0 acesso à informação amplia a capacidade de argumentação dos sujeitos políticos coletivos nos processos decisórios, ou seja, na construção do Pacto pela Democratização e Qualidade da Comunicação e Informação em Saúde. Isso implica a possibilidade dos consel hos de saúde deliberar sobre a política de informação e comunicação em saúde e intervir na realidade hoje existente. De acordo com $\mathrm{M}$ oraes $^{11}$, a gestão da informação, inclusive da saúde, permanece nas mãos dos que entendem, dos que sabem, enfim dos técnicos.

O Conselho Nacional de Saúde, através da Comissão Intersetorial de Comunicação e Informação em Saúde e da realização dos Seminários Regionais ${ }^{12}$ e Nacional, tem identificado as ten- dências do controle social para a construção do Pacto pela Democratização e Qualidade da Comunicação e Informação em Saúde para o exercício do controle social, entre as quais destacamos: 1) O Sistema Ú nico de Saúde é uma conquista da população, mas ainda não atende inúmeros problemas de saúde existentes no Brasil. Para seu avanço, se faz necessário democratizar e melhorar a qualidade da comunicação e informação em saúde; 2) a democratização e a qualidade da comunicação e informação em saúde fazem parte da luta da sociedade brasileira em seu caminhar por um país cada vez mais equânime que propicie, para todos, condições de vida e saúde dignas; 3) o Conselho N acional de Saúde constituiu a Comissão Intersetorial de Comunicação e Informação em Saúde. Essa Comissão tem como objetivo a busca pela ampliação da participação da sociedade na definição de PN$\mathrm{CIS}$; 4) quais estratégias devem ser traçadas para comunicar as informações relevantes? Essa éuma questão central que esteve presente nos debates. Os participantes buscaram alcançar consensos possíveis do que é relevante comunicar e informar, como também no que diz respeito a como comunicar e informar adequadamente; 5) no caso dos conselhos de saúde, estamos diante da necessidade de aprimoramento do que vem sendo chamado de comunicação pública, ou seja, um conjunto de ações envolvendo ou não meios técnicos de comunicação, que pressupõe o reconhecimento do amplo campo de comunicação, onde as políticas de saúde são construídas e disputadas como consensos na dimensão pública; 6) a informação não é suficiente, mas necessária para a construção da emancipação e para a apropriação da informação em saúde pelos conseIhos e demais sujeitos partícipes do debate em torno da saúde. A informação contribui para ampliar a capacidade de argumentação dos sujeitos políticos na defesa de seus interesses nos fóruns definidores da política de saúde e, como parte integrante dessa, da política de Informação e Comunicação em Saúde, que se torna assim, espaço estratégico de luta emancipatória; 7) o processo de sucateamento das instâncias públicas de gestão da informação e informática em saúde, fortalecendo a tendência pela opção da terceirização; 8) a dificuldade no processo de apropriação da informação em saúde pelos conselhos. Ter acesso à informação sem apropriarse de seu significado, sentido e contexto empobrece o potencial de uso da informação nas discussões sobreas políticas de saúde; 9) as decisões relacionadas à informação em saúde são toma- 
das com baixa participação dos conselhos; 10) a disseminação da informação em linguagem inadequada à população e consel heiros, sendo necessário decodificar a linguagem técnica para uma linguagem próxima do universo conhecido pelos conselheiros e; 11) a fragmentação dos sistemas de informações, com gestão pulverizada nas instituições de saúde.

No Seminário Nacional de Comunicação, Informação e Informática ${ }^{13}$, realizado em 8 e 9 de dezembro de 2005, identificam-se várias reivindicações do controle social para o seu fortalecimento, das quais se destacam: a divulgação do SUS; 0 esclarecimento para a sociedade do que é o controle social; a implementação das deliberações dos conselhos de saúde; a utilização de formas de comunicação e informação alternativas mais acessíveis à população; a criação de canais permanentes de comunicação e informação para dar visibilidade às deci sões dos consel hos de saúde; a clareza nas informações prestadas pelo gestor às instâncias de controle social; a condição de acesso ao orçamento e garantia da prestação de contas por parte do executivo; a disponibilização para a sociedade de informações em saúde pela mídia sobre ações e serviços de saúde, a divulgação de experiências bem-sucedidas do SU S e orientação a sociedade sobre 0 direito à saúde; garantia aos consel hos desaúde de acesso aos equipamentos de informática; a realização da Conferência de Informação, Comunicação e Informática em Saúde; a democratização da informação e da comunicação; 0 tratamento da informação e comunicação como questão estratégica, destinando recursos para a aquisição de equipamentos e capacitação dos conselheiros de saúde para a apropriação de tecnologias necessárias; 0 incentivo à formação das comissões de comunicação e informação em saúde no âmbito dos conselhos estaduais e municipais; a inclusão de participação de outros ministérios einstituições com vistas a garantir uma visão intersetorial; 0 acompanhamento e monitoramento constante do
Cadastro Nacional de Conselhos de Saúde; a opção pelo software livre no processo de informatização do SUS. Caberáao CN S propiciar uma capacitação para os conselheiros de saúde com conteúdos que contemplem a informação, comunicação e informática em saúde com responsabilidade nas três esferas de governo e viabilizar um projeto deinclusão digital com acesso a equipamentos para o uso e apropriação de novos conhecimentos em saúde.

\section{Considerações finais}

Cabe afirmar que pensar na relação controle social e a construção de uma política de informação e comunicação em saúde significa que temos que avançar no processo de democratização que vem se imprimindo na realidade brasileira. Isso implica a redistribuição efetiva do poder e do saber. Considera-se um avanço a instituição da Comissão Intersetorial de Comunicação eInformação em Saúde no âmbito do Conselho Nacional de Saúde, que tem proporcionado a ampliação desse debate. Essa iniciativa tem fomentado a criação de comissões similares nos conselhos estaduais e municipais de saúde. $\mathrm{Na} 12$ Conferência Nacional de Saúde, foi avaliado que, no cenário mundial e no Brasil, as políticas e estratégias setoriais de comunicação e informação encontram-se no eixo central das possibilidades de geração de novos processos e de mudanças nos modelos institucionais de gestão. Para tanto, sefaz necessário democratizar a comunicação ea informação e garantir a implementação de uma política de comunicação e informação em saúde no Conselho Nacional de Saúde, fortalecido e articulado com os consel hos estaduais e municipais, no sentido de ampliar a comunicação destes com o governo e a sociedade. Isso consiste num desafio para o exercício do controle social no sentido de garantir a democratização, a apropriação do acesso e o direito à saúde. 


\section{Colaboradores}

Silva AX, Cruz EA, M elo V participaram igualmente de todas as etapas da elaboração do artigo.

\section{Referências}

1. Lei $n 08.080$ de 19 de setembro de 1990. Dispõe sobre as condições para a promoção, proteção e recuperação da saúde, a organização e o funcionamento dos serviços correspondentes e dá outras providências. Diário Oficial da União 1990; 19 set.

2. Lei no 8.142 de 28 de dezembro de 1990. Dispõe sobre a participação da comunidade na gestão do Sistema Único de Saúde e sobre as transferências intergovernamentais de recursos financeiros na área da saúde e dá outras providências. Diário Oficial da União 1990; 31 dez.

3. Chauí MS. Cultura e democracia: o discurso competente e outras falas. 6a Ed. São Paulo: Cortez; 1993.

4. Ministério da Saúde. 12 a Conferência Nacional de Saúde - Sérgio Arouca. Brasília, dez. 2005.

5. Cardoso JM. Comunicação e Saúde: Desafios para fortalecer o SUS, ampliar participação e o controle social. In: Coletânea de Comunicação e Informação em Saúde para o exercício do Controle Social. Braślia: Editora do Ministério da Saúde; 2006. p. 45-56.

6. Oliveira CO. Desafios e contradições comunicacionais nos conselhos de saúde. In: Coletânea de Comunicação e Informação em Saúde para o exercício do Controle Social. Brasília: Editora do Ministério da Saúde: 2006. p. 29-46.

7. Ministério da Saúde. 10 a Conferência Nacional de Saúde. Brasília, set. 1996.

8. Ministério da Saúde. 11a Conferência Nacional de Saúde. Brasília, dez. 2000
9. Lelis GD, Rezende MTMC, Baumgarten MH, Moraes IHS, Santos GA, Silva AX, et al. Plano de trabaIho da Comissão Intersetorial de Comunicação e Informação em Saúde do Conselho Nacional de Saúde Brasília: M inistério da Saúde/Conselho Nacional de Saúde; 2005.

10. M oraes IHS. Informação em saúde para o fortalecimento do controle social do SUS. In: Seminário de Comunicação, Informação e Informática em Saúde. Braślia: Editora do Ministério da Saúde; 2005. p. 31-42.

11. Moraes IHS. Política Nacional de Informação, Informática e Comunicação em Saúde: um pacto a ser construído. Revista Saúde em Debate 2005; 29(69):86-98.

12. Conselho Nacional de Saúde. Seminários Regionais de Comunicação, Informação e Informática em Saúde. Brasília: Editora do Ministério da Saúde; 2005.

13. Conselho Nacional de Saúde. Seminário Nacional de Comunicação, Informação e Informática em Saúde - Pacto pela Democratização e Qualidade da Comunicação e Informação em Saúde. Brasília: Ministério da Saúde. No prelo 2006.

Artigo apresentado em 29/06/2006

Aprovado em 24/11/2006

Versão final apresentada em 14/12/2006 